\title{
To be or not to be? Lebensdynamiken internationaler Organisationen im Spannungsfeld von internationaler Autorität und nationalstaatlicher Souveränität ${ }^{1}$
}

Die Forschung zu internationalen Organisationen (IOs), die sich bisher überwiegend mit der Entstehung und dem Design von IOs beschäftigte, steht aufgrund des Brexits und der Ankündigung verschiedener Staaten aus dem Internationalen Strafgerichtshof auszutreten vor neuen Herausforderungen. Austritte von Mitgliedsstaaten sind nur eine Form von staatlichem Handeln, das die Autorität von IOs beeinträchtigt. Dieser Beitrag argumentiert, dass neben Austritten auch Budgetkürzungen, Personalblockaden, Mandatsbeschränkungen und die Verletzung zentraler Normen IOs unter Druck setzen. Die zentrale Frage hierbei ist, wie IOs selbst mit diesen Herausforderungen umgehen. Basierend auf Theorien der Multilateralismus- und der Organisationsforschung analysiert dieser Beitrag Nicht-Reaktion, Vermeiden von Aufmerksamkeit, Anpassung und Resilienz-Bildung. Diese Herausforderungen und Reaktionen werden anhand des historischen Beispiels des Völkerbundes illustriert. Abschließend werden mögliche Annahmen hinsichtlich des institutionellen Designs von IOs, der Art der Herausforderung, des Status des herausfordernden Staates und der Positionen der übrigen Mitgliedsstaaten diskutiert, um die Varianz in den Reaktionen von IOs zu erklären.

\section{Einleitung}

Internationale Organisationen (IOs) sind aus der heutigen zwischenstaatlichen Kooperation und dem System von global governance nicht mehr wegzudenken. Die Ausweitung ihres Einflussbereichs bei gleichzeitigen Legitimitätsdefiziten und Infragestellung ihrer Kompetenz ruft jedoch zunehmenden Widerstand hervor (Zürn 2018b). Dieser Widerstand äußert sich zum einen in stärkerer gesellschaftlicher Politisierung (Seibel 2015; Zürn/Ecker-Ehrhardt 2013; Zürn et al. 2012), zum anderen auch immer mehr darin, dass einzelne, auch einflussreiche Mitgliedsstaaten für sich in Anspruch nehmen, durch bestimmte Handlungen ihre Souveränität zurückzufordern (Alter et al. 2016; Borzyskowski/Vabulas 2019; Helfer 2005; Jones 2018; Pauwelyn/Hamilton 2018; Soley/Steininger 2018). Der Brexit ist in Europa das bekann-

1 Dieser Beitrag beruht auf dem von der Autorin geleiteten Projekt „Multilateralism under attack“, gefördert von der Fritz-Thyssen-Stiftung, und wurde zur Tagung „Die Zukunft des globalen Regierens" vom 17.-18.5.2019 in der Politischen Akademie Tutzing anlässlich des 60. Geburtstags von Michael Zürn verfasst. Für hilfreiche Kommentare bedanke ich mich herzlich bei Martin Binder, Inken von Borzyskowski, Mette Eilstrup-Sangiovanni, Benjamin Faude, Monika Heupel, Tobias Lenz, Christian Rauh und Stefanie Walter sowie bei den anonymen GutachterInnen. 
teste aktuelle Beispiel, doch auch der Internationale Strafgerichtshof ist mit Austritten oder Austrittsdrohungen unter Inanspruchnahme von Souveränität konfrontiert. ${ }^{2}$ Darüber hinaus kürzen wichtige Mitgliedsstaaten wie die USA ihre Budgets für IOs oder blockieren die Ernennung von Schlüsselposten, wie z.B. der RichterInnen des Streitbeilegungsmechanismus' der Welthandelsorganisation (WTO). ${ }^{3}$ Weitere Staaten weigern sich systematisch zentrale Kernnormen bestimmter Organisationen einzuhalten, so wie beispielsweise Polen und Ungarn das Rechtsstaatsprinzip der Europäischen Union (EU) verletzen (Magen 2016). ${ }^{4}$

Diese staatlichen Handlungen bedrohen den Kern der Arbeitsweise und mittelfristig womöglich sogar die Existenz von IOs, weshalb aktuell mehrfach von einer Krise des Multilateralismus oder dem Niedergang der liberalen Ordnung gesprochen wird (Ikenberry 2018). ${ }^{5}$ Nun war das Verhältnis zwischen Staaten und den von ihnen gegründeten IOs von Anfang an konfliktbeladen. Seit der Gründung von IOs ist dieses Verhältnis durch einen grundsätzlichen Konflikt gekennzeichnet, der zwischen dem kurzfristigen Interesse von StaatenvertreterInnen am Machterhalt und dem eher langfristigen Mehrwert multilateraler Kooperation besteht. Auch Austritte aus IOs sind keineswegs ein neues Phänomen (Borzyskowski/Vabulas 2019). Bemerkenswert ist aber, dass dieses Handeln von Staaten aktuell oftmals mit der Rückforderung von Souveränität begründet wird. Nationalstaatliche Souveränität wird von Staaten instrumentalisiert und als Antithese gegen IOs und multilaterale Kooperation verwendet. StaatenvertreterInnen argumentieren hierbei, dass die Mitgliedschaft in IOs sie in ihrer Souveränität beschneiden würde und sie dementsprechend entweder den Einfluss von IOs zurückschneiden oder Konsequenzen hinsichtlich ihrer Mitgliedschaft zu ziehen hätten.

Dieses auf das Nationale begrenzte Souveränitätsverständnis steht im Widerspruch zu einem nach außen gerichteten Souveränitätsgedanken, wonach die Mitgliedschaft in IOs eine Bestätigung der gegenseitigen Anerkennung staatlicher Souveränität ausdrückt und die Existenz von IOs selbst ein Ausdruck staatlicher Souveränität ist. Staaten scheinen hierbei die externe Anerkennung durch die internationale Staatengemeinschaft, ein zentraler Bestandteil staatlicher Souveränität seit 1945 (Zürn/Deitelhoff 2015; Chayes/Handler Chayes 1995), als weniger relevant zu betrachten. Kurz gesagt: während zwischenstaatliche Kooperation innerhalb von IOs bisher zentraler Bestandteil des Selbstverständnisses eines sich als international

2 Agence France-Presse (AFP) 2016. 'Don't go!' ICC officials appeal to African defectors, The East African, 17 November 2016 (http://www.theeastafrican.co.ke/news/Do-not-go-IC C-officials-appeal-to-African-defectors--/2558-3455402-ea4aau/index.html; 23.8.2019).

3 British Broadcasting Cooperation (BBC) 2017. US Withdraws Funding for United Nations Population Fund, 4 April 2017 (https://www.bbc.com/news/world-us-canada-39487617; 23.8.2019); Miles, Tom 2018: U.S. blocks WTO judge reappointment as dispute settlement crisis looms, Reuters, 27 August 2018.

4 Kroet, Cynthia 2017. Judicial plans would remove Poland's safety valve warns ombudsman, Politico, 23 October 2017 (https://www.politico.eu/article/judicial-plans-would-remo ve-polands-safety-valve-warns-ombudsman/; 10.1.2018).

5 Dworkin, Anthony and Richard Gowan 2019: Rescuing multilateralism, Policy Brief, 25.7.2019, European Council on Foreign Relations (https://www.ecfr.eu/publications/sum mary/rescuing_multilateralism; 23.8.2019). 
souverän betrachtenden Staates war, wird das Konzept der nationalstaatlichen Souveränität aktuell dazu verwendet ebendiese Kooperation (zumindest in Teilen) aufzukündigen. Insbesondere seit 2016 werden diese sich widersprechenden Souveränitätsverständnisse vermehrt von Staaten gegeneinander gestellt. Dadurch eröffnet sich eine neue Kluft zwischen einerseits pro- und andererseits anti-multilateral eingestellten Staaten. Dies deutet auf einen grundlegenden Wandel im Souveränitätsdenken von Staaten hin und weist Anzeichen einer neuen Blockbildung auf, die für die Zukunft von global governance möglicherweise weitreichende Konsequenzen haben wird. ${ }^{6}$

Die gestiegene Autorität von IOs (Hooghe et al. 2017) hat über die letzten Jahre durchaus zu diesem gewachsenen Spannungsfeld zwischen autonomen IOs und der nationalen Selbstbestimmung von Staaten beigetragen. Die ökonomischen Sparmaßnahmen, die von der sogenannten Troika aus Europäischer Kommission, Europäischer Zentralbank und Internationalem Währungsfonds in einigen süd- und osteuropäischen Ländern als Bedingung an finanzielle Kredite geknüpft wurde, sind nur ein Beispiel für die Auswirkungen gewachsener Autorität von IOs (FischerLescano 2014; Hirschmann 2020). Auch die unabhängige Ermittlungstätigkeit des Internationalen Strafgerichtshofs wird unter dem Gesichtspunkt des Prinzips der Komplementarität, wonach nationale Rechtswege zuerst ausgeschöpft werden müssen, immer häufiger auch als Ausweitung von Autorität verstanden (Stahn/El Zeidy 2011). Aus völkerrechtlicher Sicht gibt es daher durchaus legitime Mittel für Staaten, die Autorität von IOs einzugrenzen und ihre Souveränität zurückzufordern (Sarooshi 2005; Wouters/Odermatt 2016).

Dieses empirische Phänomen findet bisher allerdings kaum systematische Beachtung in der bisherigen politikwissenschaftlichen IO-Forschung (mit Ausnahme von Gray 2018; Borzyskowski/Vabulas 2019). Deshalb ist das zentrale Anliegen dieses Beitrags, eine neue Forschungsagenda aufzuzeigen und einen konzeptionellen Rahmen hierfür zu bieten. Ziel ist es, erstens, ein Analyseraster staatlicher Handlungen, die sich gegen IOs wenden, zu entwickeln und, zweitens, mögliche Reaktionen von IOs darauf aufzuzeigen. Darüber hinaus wird diese Typologie mit aktuellen und historischen Beispielen illustriert, mit besonderem Augenmerk auf den Entwicklungen des Völkerbunds zwischen 1920 und 1939. Abschließend werden erste Hypothesen generiert, die die Unterschiede in den Reaktionen verschiedener IOs auf die staatlichen Herausforderungen erklären könnten.

Vor dem Hintergrund der oben geschilderten Schattenseiten im Leben von IOs gebe ich im Folgenden zunächst einen Überblick über die bestehende IO-Literatur, um die Notwendigkeit einer neuen Forschungsagenda aufzuzeigen. Danach konzeptionalisiere ich, teilweise basierend auf der völkerrechtlichen IO-Forschung, eine Typologie staatlicher Herausforderungen gegenüber IOs, die Budgetkürzungen, Personalblockaden, Mandatsbeschränkungen, die Verletzung zentraler Normen

6 Auf Initiative der deutschen und französischen Regierungen wurde im September 2019 bei der Generalversammlung der Vereinten Nationen offiziell eine „Allianz für den Multilateralismus" gegründet (https://www.france24.com/en/20190402-france-germany-seek-boostmultilateralism; 23.8.2019). 
und Austritte umfasst. Darauffolgend entwickele ich auf der Basis der Literatur zu IO-Bürokratien eine Typologie unterschiedlicher Reaktionen von IOs auf die staatlichen Herausforderungen, von Nicht-Reaktion über das Vermeiden von Aufmerksamkeit, Anpassung bis hin zu Resilienz-Bildung. Diese beiden Typologien werden mit Blick auf den Völkerbund kurz illustriert. Abschließend werden mögliche Annahmen hinsichtlich des institutionellen Designs von IOs, Art der Herausforderung, Status des herausfordernden Staates und Positionen der übrigen Mitgliedsstaaten diskutiert, die die unterschiedlichen Reaktionen von IOs erklären können. Der Beitrag schließt mit einem Ausblick auf die Implikationen für die IO-Forschung und die aktuelle multilaterale Praxis.

\section{Lücken in der IO-Forschung: die Notwendigkeit einer neuen Forschungsagenda}

Die Multilateralismus-Forschung zeigt, dass staatlicher Widerstand gegen IOs keineswegs neu, sondern Kernbestandteil der Lebensdynamiken von IOs ist. Bereits US-Präsident Richard Nixon äußerte sich beispielsweise mehrfach abschätzig über die Vereinten Nationen (UN):

„What is it anyway? It's a damned debating society. What good does it do? Very little [unintelligible]. They talk about hijacking, drugs, the challenges of modern society, and the rest of it is to give hell to the United States" (Keefer 2005).

Nixon war der erste US-Präsident, der es verstand, den innerstaatlichen Widerstand gegen die UN politisch und wahltaktisch auszunutzen (Keefer 2005: 16). Unter seiner und Präsident Ronald Reagans Administration wurden die US-Beiträge an die UN erheblich gekürzt; darüber hinaus traten die USA vorübergehend aus der International Labour Organization (ILO) und der United Nations Educational, Scientific and Cultural Organization (UNESCO) aus und lehnten eine Anerkennung der Rechtsprechung des Internationalen Gerichtshofs ab (Francioni 2000). Die Forschung in den Internationalen Beziehungen (IB) zum Verhältnis zwischen IOs und den USA zeigt, dass IOs immer wieder durch unilaterale Aktionen der USA herausgefordert werden (Karns/Mingst 1990b; Malone/Kong 2003). Diese Krisenmomente des Multilateralismus beschränken sich nicht auf den prominenten Bereich Sicherheit, in dem die US-Invasion im Irak 2003 letztmalig den UN-Sicherheitsrat als multilaterale Institution in Frage stellte, sondern betreffen alle Politikbereiche, über Handel, Klima bis hin zu Menschenrechten (Newman et al. 2006). Das Verhältnis zwischen den USA und IOs war von Beginn an durch eine starke Ambivalenz gekennzeichnet: während viele IOs auf Bestreben der USA gegründet und nach USVorschlägen ausgestaltet wurden, gab es immer wieder Fälle, in denen die USA einer Vertiefung der multilateralen Ordnung entgegenstanden (Luck 1999).

Spätestens das Beispiel Brexit jedoch zeigt: Die USA sind nicht der einzige Staat, der IOs herausfordert (Jones 2018; Webber 2014). ${ }^{7}$ Darüber hinaus weist auch die

7 Walter 2019: The Mass Politics of International Disintegration, Working Paper (http://www .stefaniewalter.de/publications/; 23.8.2019). 
Forschung zu rising powers darauf hin, dass Machtverschiebungen innerhalb der Mitgliedsstaaten die Entscheidungsfindung innerhalb von IOs beeinflussen (Ikenberry 2009; Zangl et al. 2016). Als Antwort auf den aktuellen Rückzug der USA wird erwartet, dass China in IOs als neue Führungskraft auftreten und die Spielregeln der Kooperation entsprechend prägen wird. ${ }^{8}$ Die Forschung zu Fragmentierung und Regime-Komplexen zeigt außerdem, wie Staaten verschiedene multilaterale Institutionen nutzen oder gegeneinander ausspielen (Gehring/Faude 2014; Morse/Keohane 2014). Trotz dieser Erkenntnisse bleibt die Frage, wie das Handeln von verschiedenen Staaten, das die multilaterale Kooperation im Kontext von IOs in Teilen oder vollständig in Frage stellt, systematisch zu analysieren ist.

Die Forschung zu IOs wird weniger von Fragen des Niedergangs als von der Analyse der Entstehung von IOs dominiert. Rationalistische Ansätze fokussieren sich auf die Fragen, wie Staaten kollektive Handlungsprobleme überwinden (Snidal 1985; Stein 1983) und warum Staaten bereit sind, formalisierten IOs beizutreten (Abbott/Snidal 1998; Koremenos et al. 2001). Konstruktivistische Ansätze betonen die Rolle von like-minded Koalitionen und gesellschaftlicher Mobilisierung für die Entstehung von IOs, exemplarisch verdeutlicht bei der Errichtung des Internationalen Strafgerichtshofs. Beiden Ansätzen ist gemein, dass in den Analysen der Aufstieg und die Ausweitung der Kompetenzen von IOs im Vordergrund steht und der Niedergang von IOs, mit wenigen Ausnahmen (Gray 2018; Koremenos 2016), bisher keine prominente Rolle spielte.

Die Zürnsche Forschung zu IOs, größtenteils unternommen durch die Abteilung Global Governance (vormals Transnationale Konflikte und Internationale Institutionen) am Wissenschaftszentrum Berlin (WZB) unter der Leitung von Michael Zürn, beschäftigt sich hauptsächlich mit der Wirkung und den Legitimitätsdefiziten von IOs (Zürn 2018b). Als aktiver Teil von global governance hat die Autorität von IOs seit 1945 signifikant zugenommen (Hooghe et al. 2017). Gleichzeitig stellen Mängel in den institutionellen Strukturen sowie Fehlverhalten und Handlungsunfähigkeit die Legitimität von IOs von Grund auf in Frage (Heupel/Zürn 2017; Tallberg/Zürn 2019). Als Konsequenz kommt es zu einer Politisierung in Teilen der (westlichen) Gesellschaft (Zürn 2018a; Zürn et al. 2012). Dies kann letztendlich Regierungen dazu veranlassen, aus IOs auszutreten, wie die aktuelle Forschung zu Referenden und Austritten zeigt. ${ }^{9}$

In der internationalen IO-Forschung wird einerseits die positive Wirkung von IOs auf zwischenstaatliche Konflikte und Kooperation in den Bereichen Handel und Umwelt hervorgehoben und ihre Rolle als zentraler Akteur zur Lösung globaler Probleme in der Interaktion mit anderen Akteuren im Bereich von global governance betont (Park 2018; Abbott et al. 2015; Tallberg et al. 2013). Auch bei

8 Gramer, Robbie and Colin Lynch 2019: Haley tried to block appointment of Chinese diplomat to key U.N. post, Foreign Policy 14.2.2019 (https://foreignpolicy.com/2019/02/14/unit ed-nations-china-xia-huang-influence-africa-great-lakes-diplomacy-nikki-haley-united-stat es-international-organizations/; 23.8.2019).

9 Walter 2019: The Mass Politics of International Disintegration, Working Paper (http://www .stefaniewalter.de/publications/; 23.8.2019). 
der Entwicklung von Normen und der Überwachung ihrer Einhaltung durch Mitgliedsstaaten wird IOs eine wichtige Rolle zugeschrieben (Finnemore/Sikkink 1998). Andererseits aber gerieten durch den gewachsenen Einfluss der bürokratietheoretischen Forschung in den letzten Jahren auch immer mehr die Defizite und Probleme von IOs in den Blick: Pathologien (Barnett/Finnemore 2004; Dijkzeul/ Beigbeder 2003), Agency-Probleme (Graham 2014), organized hypocrisy (Hirschmann 2012; Lipson 2007), die Ausweitung der Kompetenzen ohne die Zustimmung der Mitgliedsstaaten (Johnson 2014), Accountability-Probleme (da ConceiçãoHeldt 2016; Hirschmann 2019) - all dies sind wichtige Aspekte der Entwicklung von IOs, die teilweise für den Rückzug von Staaten aus IOs verantwortlich sind.

Die Forschung in IB und Völkerrecht weist auf Wege hin, wie Staaten Kontrolle über IOs zurückgewinnen und deren Autorität einschränken (könnten) (Hawkins et al. 2006; Sarooshi 2005; Wouters/Odermatt 2016). Darüber hinaus zeigt die neueste Forschung, dass IOs weniger robust sind, als bisher angenommen: nicht alle jemals gegründeten IOs haben überlebt. Im Gegenteil, gut ein Drittel der 561 IOs, die nach 1815 entstanden, haben bis 2015 aufgehört zu existieren. Diese wurden entweder geschlossen oder existieren komatös oder als Papierexistenzen fort (Gray 2018; Eilstrup-Sangiovanni 2018). Angesichts dieser Zahlen ist es höchste Zeit zu untersuchen, wie IOs selbst mit der Rückforderung nationaler Souveränität und der Einschränkung ihrer Autorität durch Mitgliedsstaaten umgehen. Im Folgenden soll dieser Artikel einen Beitrag zur Beantwortung dieser zentralen Frage leisten, indem zunächst ein konzeptioneller Rahmen für die Analyse von staatlichem Handeln sowie der Reaktionen von IOs darauf entwickelt werden.

\section{Die Rückforderung staatlicher Souveränität: eine Typologie}

IOs charakterisieren sich durch ihre finanzielle und personelle Ausstattung, ihre Mandatsstruktur, ihre Konstitution durch Mitgliedsstaaten sowie die Implementierung ihrer Politiken (Sarooshi 2005; Wouters/Odermatt 2016). Staaten nehmen auf zahlreichen Wegen Einfluss auf IOs; jedoch argumentiere ich, dass alles staatliche Handeln, das die oben genannten Elemente limitiert oder verhindert, die Autorität von IOs einschränkt und potenzielle Reaktionen erwarten lässt. Daraus ergibt sich folgende Typologie als Analyseraster von staatlichem Handeln vis-à-vis IOs, illustriert durch jeweils aktuelle und historische Beispiele.

Erstens, Budgetkürzungen: IOs sind daran gewöhnt, dass Staaten ihre Mitgliedsbeiträge mit teilweise erheblicher zeitlicher Verzögerung zahlen. Oftmals steht dies in Verbindung mit Forderungen nach institutionellen Reformen. Sogenannte earmarked contributions wurden in den letzten zwei Jahrzehnten zu einem populären Mittel, womit Staaten finanzielle Zuwendungen an IOs an bestimmte inhaltliche Politikausrichtungen knüpfen (Graham 2015). Auch wenn dieses Verhalten völkerrechtlich gesehen durchaus legal ist, führt dies zu einer Bedrohung der Unabhängigkeit und Legitimität von IOs und ist damit indirekt existenzgefährdend. Direkt existenzbedrohend wird es jedoch für IOs, wenn finanziell beitragsstarke Staaten 
sich weigern, ihre Mitgliedsbeiträge zu zahlen oder diese signifikant reduzieren. Dies kann als Zeichen einer Ablehnung von multilateraler Kooperation gedeutet werden, aber auch eine Konsequenz von wirtschaftlichen Bedingungen sein, wie z.B. der globalen Finanzkrise von 2008. Unabhängig von der Motivation hat dies jedoch unmittelbar Auswirkungen auf die Autorität und Funktionsweise von IOs.

Das Verhalten der USA als finanzstärkster Beitragszahler ist hierbei von besonderer Bedeutung. Weigerungen der US-Regierung den Beitrag zum Budget von UN-Friedensoperationen oder zu einzelnen UN-Organisationen wie den UN Populations Fund (UNFPA) zu zahlen, haben starke Auswirkungen auf die Handlungsfähigkeit dieser Organisationen. IOs, deren Budgets sich aus hauptsächlich freiwilligen Beitragszahlungen eines einzelnen Staates zusammensetzen, sind hierbei besonders verwundbar. ${ }^{10}$

Zweitens, Personalblockade: Die Funktionsweise von IOs beruht auf DiplomatInnen, die von Mitgliedsstaaten in die jeweiligen Gremien entsandt werden, sowie auf der Ernennung hochrangiger IO-Angestellter. Die Beschlussfähigkeit von IOGremien und die Handlungsfähigkeit der gesamten IO-Administration hängen von dieser Ernennung ab. Eine länger als sechs Monate andauernde Nicht-Entsendung nationaler RepräsentantInnen in IOs durch einen Staat kann die Entscheidungsfähigkeit und damit die Autorität von IOs fundamental einschränken. Die Blockade der Ernennung von zentralen IO-EntscheidungsträgerInnen wiederum kann an bestimmte Bedingungen geknüpft sein oder ein Zeichen der Ablehnung des entsprechenden Gremiums sein. Dies hat direkte Auswirkungen auf die Funktionsfähigkeit und mittelfristig auch auf die Existenz von IOs. Fehlende RepräsentantInnen im UN-Sicherheitsrat beispielsweise können als Veto interpretiert werden, auch wenn die Anwesenheit für die Annahme einer Resolution formal nicht nötig ist. ${ }^{11}$ Andere Gremien, wie z.B. das Streitschlichtungsverfahren der WTO, bedingen eine bestimmte Anzahl ernannter RichterInnen um die Beschlussfähigkeit zu gewährleisten.

In der Geschichte von IOs finden sich zahlreiche Beispiele, wie Staaten Personalblockaden eingesetzt oder die Absetzung von einflussreichem Personal vorangetrieben haben. Eines der aktuellsten Beispiele ist die Blockade der US-Regierung, neue RichterInnen für den Streichschlichtungsmechanismus der WTO zu ernennen, was den Mechanismus seit Dezember 2019 beschlussunfähig lässt. ${ }^{12}$ Zuvor wandte die US-Regierung Personalblockaden insbesondere im Rahmen der Weltbank an (Hunter Wade 2002). Das Bespiel Regionalorganisationen zeigt außerdem, dass dieses Phänomen keineswegs nur auf die USA beschränkt ist: Kenia versuchte beispielsweise RichterInnen am East African Court of Justice abzusetzen, nachdem der Ge-

10 BBC 2017. US Withdraws Funding for United Nations Population Fund, 4 April 2017 (https://www.bbc.com/news/world-us-canada-39487617; 19.2.2020).

11 Die Nicht-Ernennung eines US-Botschafters bei den UN wurde z.B. als Begründung angeführt, warum eine Resolution als Antwort auf eine Attacke auf Flüchtlingslager wegen einer fehlenden US-Position nicht verabschiedet werden konnte.

12 Walker, Andrew 2019: Trade disputes settlement system facing crisis, BBC, 8.12.2019, (https://www.bbc.com/news/business-50681431; 10.1.2020). 


\section{Aufsätze}

richtshof entgegen der vorgebrachten nationalen Interessen Kenias entschieden hatte (Alter et al. 2016).

Drittens, Mandatsbeschränkung: Bei der Einrichtung einer IO verständigen sich die Gründungsstaaten auf ein Mandat für diese IO. Bestehende Analysen hinsichtlich der Autorität von IOs zeigen, dass diese Mandate stark variieren, von einem begrenzten bis zu einem äußerst umfassenden Kompetenzspektrum (Hooghe et al. 2017). Zunehmende Autorität kann bedeuten, dass IOs dieses Mandat ohne Zustimmung ihrer Mitgliedsstaaten in den Jahren nach der Gründung signifikant ausweiten (Johnson 2014). Die Autorität von IOs wird dementsprechend eingeschränkt, wenn diese Mandate von Mitgliedsstaaten begrenzt oder sogar beendet werden. Eine Beendigung aufgrund von Mandatserfüllung wird in dieser Typologie jedoch außen vorgelassen, auch wenn dies zu einer Auflösung einer IO führen kann, da dies dem Erfolg einer IO und nicht einer Ablehnung auf Seiten der Mitgliedsstaaten zugeschrieben werden kann.

Aktuell sind Fälle von Mandatsbeschränkungen besonders bei internationalen Gerichten zu finden. Zimbabwe beispielsweise führte erfolgreich eine Initiative an, dem Tribunal der South African Development Cooperation (SADC) das Recht zu nehmen, Beschwerden von privaten Individuen anzunehmen (Alter et al. 2016). Mit dieser Mandatsbeschränkung wurde die Möglichkeit der Rechtsprechung dieses Tribunals stark eingeschränkt. In einem anderen Fall war der Versuch einer Mandatsbeschränkung nicht erfolgreich: Gambia scheiterte daran, dem Gericht der Westafrikanischen Wirtschaftsgemeinschaft (ECOWAS) das Mandat abzusprechen, über Menschenrechtsverletzungen zu urteilen (Alter et al. 2016). Hierbei wird sichtbar, dass einzelne einflussreiche Staaten den Prozess initiieren und forcieren können, jedoch ein Verbund von Staaten für eine erfolgreiche Mandatsbeschränkung erforderlich ist.

Viertens, systematische Nicht-Einhaltung zentraler Normen: Die Existenz von IOs beruht weiterhin darauf, dass ihre Mitgliedsstaaten sich zur Einhaltung fundamentaler Normen bekennen. Diese Normen sind in der Charta, den aktuellen Verträgen oder dem Gründungsvertrag verankert und deshalb von allen Mitgliedsstaaten unterzeichnet und ratifiziert. Der Fokus liegt hierbei auf konstitutiven Normen, die als zentral für den Charakter und die Identität der IO und den darin versammelten Mitgliedsstaaten erachtet werden. Während es in der Geschichte von IOs immer wieder zu Normverletzungen einzelner Mitgliedsstaaten kam, zeugt eine systematische Nicht-Beachtung einer zentralen Norm durch einen Mitgliedsstaat von der Ablehnung zentraler Werte, die für die Existenz dieser IO und die Ausübung ihrer Autorität von Belang sind.

Aktuellstes Beispiel hierfür ist die systematische Verletzung des Rechtsstaatsprinzips der EU durch Polen und Ungarn. Beide Länder verabschiedeten nationale Gesetze und unternahmen Politikentscheidungen hinsichtlich der Besetzung nationaler Gerichte, die im Widerspruch zu dieser zentralen EU-Norm stehen (Magen 
2016). ${ }^{13}$ Auch einzelne Normverletzungen können die Autorität von IOs stark untergraben, wenn diese von anderen Mitgliedsstaaten nicht sanktioniert, sondern im Gegenteil nachgeahmt werden.

Fünftens, Austritt: Die Existenz von IOs definiert sich zentral darüber, dass sie durch Mitgliedsstaaten konstituiert sind. Wenn ein oder mehrere Mitgliedsstaaten sich aus IOs oder einzelnen Gremien von IOs zurückziehen, hat dies unmittelbare Auswirkungen auf die Existenz von IOs, auch wenn dieser Austritt rechtlich gesehen legal sein kann (Brölmann et al. 2018). Dies geschieht durch eine formale Ankündigung des Austritts aber auch durch die längerfristige Weigerung eines Mitgliedsstaates an zentralen Gremien teilzunehmen (wie z.B. während der Krise des leeren Stuhls in den 1960ern in der Europäischen Gemeinschaft). Bisherige Analysen verweisen darauf, dass diese Art der Dis-Integration schwerwiegende Auswirkungen auf die verbleibenden Mitgliedsstaaten hat. ${ }^{14}$

Als prominentestes Beispiel hierfür wird die Entscheidung Großbritanniens aus der EU auszutreten angeführt. Insbesondere für das Budget der EU hat dieser Austritt weitreichende Konsequenzen, wie die Brexit-Verhandlungen gezeigt haben. Aber auch andere IOs wie z.B. der Internationale Strafgerichtshof sind mit Austritten oder Austrittsdrohungen konfrontiert: verschiedene Staaten der Afrikanischen Union (AU) kündigten an, ihre Mitgliedschaft zu überdenken und Burundi sowie die Philippinen haben ihre Mitgliedschaft bereits aufgekündigt. Gambia und Südafrika haben ihre angekündigten Austritte wieder rückgängig gemacht. Auch der Völkerbund war bereits in seinen Anfangsjahren mit einer Austrittswelle, insbesondere von südamerikanischen Staaten, konfrontiert, die die globale Dimension der Autorität des Völkerbunds und die Legitimität der Organisation in Frage stellte (Edwards 1929).

Diese Typologie zeigt, dass staatliches Handeln auf fünffache Weise die Autorität von IOs einschränken kann. Die hierbei zugrundeliegenden Motivationen können durchaus variieren. Die Berufung auf nationalstaatliche Souveränität scheint in der aktuellen Krise jedoch eine gewichtigere Rolle zu spielen als in den vergangenen Jahrzehnten, zumindest wenn man die Forschung zu vergangenen Austritten heranzieht (Borzyskowski/Vabulas 2019). Während sich diese Forschung bisher überwiegend auf die Gründe für ein derartiges Verhalten von Mitgliedsstaaten fokussierte, sollen im Folgenden mögliche Reaktionsmuster von IOs selbst darauf diskutiert werden.

13 Kroet, Cynthia 2017. Judicial plans would remove Poland's safety valve warns ombudsman, Politico, 23 October 2017 (https://www.politico.eu/article/judicial-plans-would-rem ove-polands-safety-valve-warns-ombudsman/; 10.1.2018).

14 Walter 2019: The Mass Politics of International Disintegration, Working Paper (http://ww w.stefaniewalter.de/publications/; 23.8.2019). 


\section{Eine Typologie von IO-Reaktionen auf die Einschränkung ihrer Autorität}

Hinsichtlich der Frage, wie IOs auf derlei Verhalten einzelner Mitgliedsstaaten reagieren, fokussiert sich die bestehende Forschung besonders auf die Reaktion der verbleibenden Mitgliedsstaaten (Alter et al. 2016; Eilstrup-Sangiovanni 2018; Gray 2019). Die Reaktion der IO-Administrationen und Bürokratien wurde jedoch bisher vernachlässigt. Vor diesem Hintergrund entwickelt dieser Beitrag eine Typologie von möglichen Reaktionen von IO-Bürokratien auf die oben geschilderten staatlichen Herausforderungen. Wichtig hierbei ist klarzustellen, dass die Grundgesamtheit der Fälle, auf die sich diese Typologie bezieht, alle IOs beinhaltet, die ein Minimum an Autorität besitzen, die von den herausfordernden Mitgliedsstaaten begrenzt wird (Hooghe et al. 2017).

Ich gehe dabei davon aus, dass IOs autonome Akteure sind und deshalb ein intrinsisches Interesse daran haben, ihre Position im multilateralen System zu bewahren (Barnett/Finnemore 2004). IOs sind komplexe Organisationen, die sowohl aus bürokratischen als auch mitgliedsstaatlichen Elemente bestehen (Biermann 2017). Reaktionen von IOs sind deshalb als Zusammenspiel der IO-Bürokratie und den anderen, „loyalen“ Mitgliedsstaaten zu analysieren. Basierend auf Erkenntnissen der Organisationsforschung (Haas 1990; Reinalda/Verbeek 2004), der völkerrechtlichen Forschung (Sarooshi 2005; Wouters/Odermatt 2016) und ersten Interviews mit IO-MitarbeiterInnen sind folgende vier Reaktionstypen zu erwarten.

Ausgangspunkt dieser Typologie ist die Option der Nichtreaktion (inertia). Eine Veränderung im Handeln der IO in unmittelbarer Reaktion auf die Herausforderung durch einzelne oder mehrere Mitgliedsstaaten ist hierbei nicht zu erkennen. Angelehnt an die Literatur zu policy inaction gehe ich davon aus, dass Nichtreaktion eine strategische Entscheidung der IO sein kann oder aber auch ein Zeichen für die Unfähigkeit der Organisation ist, institutionelle Veränderungen herbeizuführen (Bach/ Wegrich 2019; McConnell/'t Hart 2019). In einer Typologie für mögliche Reaktionen von IOs ist es wichtig zu berücksichtigen, dass IOs nicht immer willens oder in der Lage sind, auf die Einschränkung ihrer Autorität zu reagieren. Nichtreaktion kann zum einen im Zeitraum unmittelbar nach der Herausforderung geschehen oder auch einen längeren Zeitraum in Anspruch nehmen.

Die zweite Option ist eine Reaktion wodurch die IO versucht, keine weitere Aufmerksamkeit auf sich zu ziehen und sich gewissermaßen unterhalb des Radars der öffentlichen Sichtbarkeit zu bewegen (hunkering). Dies betrifft sowohl die Interaktion mit dem herausfordernden Mitgliedsstaat als auch die generelle öffentliche Wahrnehmung. Damit tritt die IO durch die Abnahme der Sichtbarkeit einer weiteren Politisierung entgegen. Die aktuelle Forschung zu IO-Kommunikation zeigt, dass IOs durchaus strategisch nach außen kommunizieren (Ecker-Ehrhardt 2018). Die völkerrechtliche Literatur identifizierte diese Strategie. ungünstige Schlagzeilen zu vermeiden. als Überlebensstrategie, besonders für internationale Gerichte und Menschenrechtsinstitutionen (Helfer 2018). Dies kann sowohl antizipierend in Reaktion auf die Herausforderung einer anderen IO im selben Bereich geschehen, als auch als Reaktion auf die eigene Herausforderung durch einen Mitgliedsstaat. 
Empirische Indikatoren dafür sind die Weigerung eine IO Presserklärungen zur umstrittenen Thematik oder zum Verhältnis mit dem herausfordernden Mitgliedsstaat herauszugeben oder eine generelle Abnahme der öffentlichen Kommunikation.

Die dritte Option ist eine Reaktion wodurch sich die IO an die Forderungen des herausfordernden Mitgliedsstaates anpasst, um dessen Unterstützung zu behalten (adaptation). Dieser Reaktionstyp basiert auf den Annahmen der Prinzipal-AgentForschung, nach denen sich IOs als Agenten von Mitgliedsstaaten nach deren Bedürfnissen richten (Hawkins et al. 2006). Anpassung kann zum einen durch die Umbenennung einzelner Politiken geschehen (z.B. von Fortpflanzungsgesundheit zu Müttergesundheit), aber auch durch die inhaltliche Veränderung oder Beendigung einzelner Programme. Beispielsweise veranlasste die Androhung der amerikanischen Reagan-Regierung signifikante Budgetkürzungen vorzunehmen einzelne IOs wie die Weltbank dazu, die Politikausrichtung und Entscheidungsfindung den US-Interessen anzupassen (Karns/Mingst 1990a: 306; Daugirdas 2015). ${ }^{15}$ Weitere Indikatoren sind institutionelle Reformen, die dem herausfordernden Staat mehr Einfluss auf die die Souveränität dieses Staates betreffenden Entscheidungen einräumen.

Die vierte Option ist eine Reaktion, wodurch die IO institutionelle Kapazitäten oder Koalitionen aufbaut, um den Herausforderungen zu widerstehen (resilience). Dies beruht auf der Forschung zur Autonomie von IOs, wonach IOs sich zunehmend von der Kontrolle durch Mitgliedsstaaten isolieren (Johnson 2014). Anstatt dies als dysfunktionales Verhalten zu klassifizieren, können institutionelle Veränderungen hin zu größerer Autonomie jedoch auch als Selbstschutz fungieren und wiederum ihre Autorität steigern (Hooghe et al. 2017). Dies kann zum einen durch bürokratische AkteurInnen geschehen (Biermann 2017; Bauer/Ege 2016). General-SekretärInnen oder PräsidentInnen spielen hierfür eine wichtige Rolle als interne NormunternehmerInnen (Gray 2019; Kraske et al. 1996). Internationale Bürokratien haben darüber hinaus Allianzen mit anderen IO-MitarbeiterInnen oder Nichtregierungsorganisationen gebildet, um Druck auf den herausfordernden Mitgliedsstaat auszuüben (Johnson 2014: 12). Durch diese Netzwerke können sie beispielsweise Staaten, die die IO verlassen wollen, an den Pranger stellen und dadurch von ihrem Vorhaben abbringen. Sie können diese Koalitionen auch nutzen, um finanzielle oder materielle Verluste auszugleichen, wie im Hinblick auf die Finanzierung des UNFPA geschehen. Gleichzeitig kann die IO-Bürokratie Allianzen mit Mitgliedsstaaten formen, die die IO gegenüber dem herausfordernden Mitgliedsstaat bestärken. Diese neuen Allianzen können normativ problematisch sein, was mit Blick auf die Rolle von China als neuer Führungskraft innerhalb der UN insbesondere im Bereich peacekeeping oder den Einfluss von privaten AkteurInnen als GeldgeberInnen im Bereich Gesundheit zu beachten ist. Empirisch ist Resilienz anhand von institutionellen Veränderungen zu beobachten, die auf neue Koalitionsbildung hinsichtlich

15 New York Times 1984: U.S. votes No in World Bank more often under Reagan, 26. November 1984, https://www.nytimes.com/1984/11/26/business/us-votes-no-at-world-bankmore-often-under-reagan.html; 11.7.2019). 
öffentlicher Unterstützung, den Aufbau alternativer finanzieller Ressourcen oder die Sanktionierung des herausfordernden Mitgliedsstaats hindeuten. ${ }^{16}$

Diese Typologie von IO-Reaktionen auf die Einschränkung ihrer Autorität durch Mitgliedsstaaten ist als Idealtyp zu verstehen. $\mathrm{Zu}$ erwarten ist, dass einzelne Typen durchaus nacheinander auftreten können. Beispielsweise versuchte die WeltbankAdministration in Reaktion auf die geplanten Budgetkürzungen der US-Administration in den 1980er Jahren zunächst Anpassung dann Koalitionsbildung, als klar wurde, dass die Reagan-Administration nicht von ihrem Vorhaben abrückte. Ein weiterer Grund für zeitlich nacheinander auftretende, verschiedene Reaktionen ist, dass inertia, hunkering und adaptation bereits nach kurzer Zeit durchgeführt werden können, während resilience oftmals einen längeren Zeitraum in Anspruch nimmt. Ein gleichzeitiges Auftreten verschiedener Reaktionstypen ist jedoch eher unwahrscheinlich: der Aufbau von institutionellem Widerstand kann nur so lange parallel zu Anpassung erfolgen, wie er vor dem herausfordernden Mitgliedsstaat verborgen bleibt.

In der aktuellen Debatte zu Brexit und anderen Herausforderungen, mit denen IOs derzeit konfrontiert sind, wird häufig auf den Völkerbund verwiesen und dafür plädiert, diesen als historisches Vergleichsbeispiel heranzuziehen, um weitere wissenschaftliche Erkenntnisse zu gewinnen (e.g. Pedersen 2007; Pedersen 2015). ${ }^{17} \mathrm{Im}$ Gegensatz zu anderen IO-Einzelfallstudien sind Untersuchungen des Völkerbunds weniger dem Vorwurf ausgesetzt, sich ausschließlich mit den in der Geschichte erfolgreichen und überlebenden IOs zu beschäftigen (Gray 2018). Mit der kürzlich begonnenen Digitalisierung des Archivs in Genf liefert der Völkerbund in der Tat Datenmaterial für einen vielversprechenden Fallvergleich, der darüber hinaus darauf hinweisen könnte, welche Art der Reaktionen für ein Überleben einer IO notwendig sind. Darüber hinaus ist der Völkerbund eine Organisation, die mit der höchstmöglichen Bandbreite an Herausforderungen konfrontiert wurde. Der Fall ist daher bestens geeignet, um die Plausibilität der Typologien zu erproben und zu illustrieren, wie unterschiedlich ein und dieselbe IO auf diese Herausforderungen reagiert. Gleichzeitig ist die Organisation ein Testfall für die Analyse von IO-Bürokratien, da im Allgemeinen angenommen wird, dass das Handeln dieser IO ausschließlich durch die Großmächte bestimmt war (Ashworth 2011). Im Folgenden sollen die oben entwickelten Typologien von Herausforderungen und Reaktionsmustern am Beispiel des Völkerbundes kurz illustriert werden. Ich beschränke mich dabei auf die Jahre 1920-1939, da der Ausbruch des Zweiten Weltkriegs überwiegend als Indikator für das Scheitern der Organisation angesehen wird.

16 In Reaktion auf die finanziellen Kürzungen der Reagan-Administration, startete die Weltbank unter Präsident Alden W. Clausen Anfang der 1980er Jahre eine öffentliche Kampagne zur internationalen Koalitionsbildung, wodurch die Finanzierung durch andere westliche Staaten schnell garantiert wurde (Kraske et al. 1996; siehe auch https://www.worldbank.org/en/about/archives/history/past-presidents/alden-winship-clausen; 11.7.2019)).

17 Cloet, Quincy 2017: Five lessons Brexit negotiators should take from the League of Nations, in: The Conversation, 11 April 2017, available at: http://theconversation.com/five-les sons-brexit-negotiators-should-take-from-the-league-of-nations-75571; 11.7.2019. 


\section{Herausforderungen und Reaktionen am Beispiel des Völkerbunds}

\section{Budgetkürzungen}

Der Völkerbund war weniger direkten Budgetkürzungen ausgesetzt, als vielmehr chronischen Zahlungsrückständen einiger Mitgliedsstaaten (sogenannte arrears). Diese Zahlungsrückstände betrafen in den Anfangsjahren überwiegend einige zentralamerikanische Staaten, wie z.B. Costa Rica sowie China. Mit dem Ausbruch der Wirtschaftskrise verschärfte sich die Situation und zahlreiche weitere Staaten, darunter Indien und Thailand, forderten erfolgreich die Reduktion ihrer Beitragszahlungen (Hell 2007: 74).

Auch wenn die Zahlungsrückstände in den meisten Fällen nicht als Ausdruck der Rückforderung nationaler Souveränität gesehen werden können, ist die Reaktion des Völkerbunds auf diese Problematik durchaus ein Beispiel innovativer Resilienz-Bildung. In den Jahren 1920-1924 wurden höhere Beitragszahlungen erhoben und ein jährlicher Überschuss in einen Fonds eingezahlt. Dieser Überschuss kompensierte die ausstehenden Beiträge und sollte eine finanzielle Notlage der Organisation verhindern. Innerhalb weniger Jahre konnte so eine solide Finanzgrundlage aufgebaut und gehalten werden, ein institutioneller Mechanismus, der für die Vereinten Nationen übernommen wurde (League of Nations 1930: 396).

\section{Personalblockade}

Im gesamten Zeitraum war der Völkerbund immer wieder damit konfrontiert, dass einzelne Mitgliedsstaaten ihre RepräsentantInnen aus den Gremien der Organisation zurückzogen. Damit nahmen diese über längere Zeit nicht an den Entscheidungen des Völkerbunds teil, ohne offiziell einen Austritt zu erklären. Argentinien war der erste Staat, der bereits nach der ersten Sitzung der Generalversammlung 1920 seinen Rückzug erklärte und bis 1933 nicht mehr teilnahm. Dies geschah, nachdem die Versammlung eine Entscheidung über verschiedene Vorschläge der argentinischen Delegation vertagte, die das Ziel hatten, eine universale Mitgliedschaft im Völkerbund zu etablieren. Im Gegensatz zu anderen Fällen war die Rückforderung nationaler Souveränität hierbei deshalb keine Grundlage (Barros 1979: 229). Anders lag es im Fall von Bolivien und Peru, die beide im Zeitraum von 1923-1929 keine Delegierten nach Genf sandten (Wehrli 2015: 2).

Die Reaktionen des Völkerbunds darauf waren eher eingeschränkt. Der Generalsekretär vermied persönliche Visiten in diesem Zusammenhang. Er befürchtete dadurch den Eindruck zu erwecken, dass der Völkerbund Druck auf die entsprechenden Mitgliedsstaaten ausüben würde. Deshalb beschränkte er sich auf diplomatische Überzeugungsarbeit und, im Falle Argentiniens, inoffizielle Besuche des britischen Botschafters in Buenos Aires (Barros 1979: 230). Das Problem potenzieller Personalblockaden innerhalb des Sekretariats wurde dadurch umgangen, dass der 
Generalsekretär, Sir Eric Drummond, bei der Besetzung zentraler Posten auf die Zustimmung der Mitgliedsstaaten achtete (Barros 1979: 391).

\section{Mandatsbeschränkung}

Auch wenn das Sekretariat gegen Ende der 1920er Jahre von den Mitgliedsstaaten für seine Unabhängigkeit kritisiert wurde (konkret: „,for being servants of the League and not its directors" (Barros 1979: 66)), unternahmen die Mitgliedsstaaten keinen Versuch, den im Vergleich zu den meisten anderen IOs sehr beschränkten Einflussbereich weiter einzuschränken. Dies kann wohl zu einem großen Teil Generalsekretär Drummond zugerechnet werden, der eine weitergehende Definierung und Ausweitung seines Mandats über den Dienst an den Mitgliedsstaaten hinaus ablehnte (Barros 1979: 50). Auch die in Art. 16 der Völkerbundsatzung festgeschriebenen Kompetenzen des Völkerbunds auf Vertragsbrüche einzelner Mitgliedsstaaten zu reagieren wurden von Drummond abgelehnt (Barros 1979: 49), weshalb eine Mandatsbeschränkung durch einzelne Mitgliedsstaaten im Fall des Völkerbunds nicht als Mittel der Rückforderung nationaler Souveränität angewandt wurde.

\section{Systematische Nicht-Einhaltung zentraler Normen}

Die Verletzung der Nicht-Angriffsnorm wurde zu einem erheblichen Problem für den Völkerbund. In den frühen Jahren forderten besonders die Angriffe Polens auf die Sowjetunion und Litauen 1920 sowie die Invasion Griechenlands in Bulgarien 1925 den Völkerbund heraus (Barros 1979: 115). In den 1930er Jahren folgten die Invasion Japans in der Mandschurei 1931/1932, die Invasion Italiens in Äthiopien 1938, die Annektierung Österreichs durch Nazi-Deutschland 1938 und die Invasion der Sowjetunion in Finnland 1939.

Die oftmals mangelhafte Reaktion des Völkerbunds auf diese wiederholten Verletzungen einer zentralen Norm wird als ein wichtiger Grund für dessen letztendliches Scheitern genannt. In der Tat reagierte der Völkerbund keineswegs konsequent auf diese Normbrüche. In den 1920er Jahren war die Androhung einer Seeblockade gegen Griechenland die stärkste Reaktion (Barros 1979: 47). Generalsekretär Drummond übte dabei maßgeblichen Einfluss aus, um diesen Konflikt auf die Agenda des Rats zu setzen (Barros 1979: 401). Das Sekretariat und die übrigen Mitgliedsstaaten bemühten sich in diesem Zusammenhang darum, eine ökonomische Waffe im Fall einer Verletzung der Nicht-Angriffsnorm im Sinne des Art. 16 zu etablieren (Barros 1979: 48). Infolgedessen wurde Anfang 1921 eine Internationale Blockade-Kommission eingerichtet, die die Einrichtung eines permanenten Sanktionierungsmechanismus analysieren und dem Rat Bericht erstatten sollte. Der Rat nahm einen wichtigen Reformvorschlag der Kommission an, nach dem Entscheidungen hinsichtlich der Sanktionierung eines Staates, der einem anderen den Krieg erklärte, mit einfacher Mehrheit getroffen werden konnten. Allerdings waren 
die Mitgliedsstaaten uneinig hinsichtlich der Frage, durch wen die Norm-Verletzung letztendlich festgestellt werden sollte. Die Beschlüsse des Rats auf der Basis dieser Berichte blieben deshalb nicht-bindend (Assembly Resolution 10; Taylor 1925: 161).

Die Normverstöße in den 1930er Jahren blieben ebenso weitgehend unsanktioniert. Ebenfalls auf Initiative des Generalsekretärs wurde eine Kommission in die Mandschurei entsandt, deren Bericht jedoch keine Sanktionen Japans nach sich zog. Italien wurde aufgrund der Invasion in Äthiopien mit Wirtschaftssanktionen belegt, allerdings ohne Wirkung, da verschiedene wirtschaftsstarke Staaten diese nicht beachteten. Parallel versuchte der Generalsekretär die USA als Partner im Sinne einer neuen Allianz gegen die normverletzenden Staaten zu gewinnen, jedoch erfolglos (Barros 1979: 51). Als eine der wenigen konkreten Sanktionen wurde die Mitgliedschaft der Sowjetunion 1939 beendet. $^{18}$

\section{Austritte}

Ein Austritt aus dem Völkerbund war durch Art. 1 der Völkerbundsatzung klar geregelt. Mitgliedsstaaten hatten die Organisation offiziell von ihrem Austrittswunsch in Kenntnis zu setzen. Der Austritt trat zwei Jahre nach dem Gesuch in Kraft, solange der Staat zu diesem Zeitpunkt seinen Verpflichtungen nachgekommen war. ${ }^{19}$ Zahlreiche Mitgliedsstaaten machten von diesem Artikel Gebrauch, was als institutionelle Schwäche des Völkerbunds identifiziert wurde. Neben diesen Austritten wird die Weigerung des US-Senats 1920 und 1921 den Vertrag zu ratifizieren als zentraler Schwachpunkt genannt.

Die Gründe für Austritte waren vielfach; der erste Staat, der sein Austrittsgesuch 1923 einreichte, war Costa Rica, dem der Völkerbund als zu teuer und wirkungslos erschien und der mit der verpflichtenden Zahlung von Mitgliedsbeiträgen nicht einverstanden war (Wehrli 2015: 2; Ellis 1928: 440). Brasilien und Spanien hingegen drohten mit und vollzogen Austritte, um einen permanenten Sitz im Rat zu verhandeln, allerdings erfolglos (Edwards 1929: 150; Barros 1979: 183). Brasilien versuchte darüber hinaus in den Verhandlungen mit Drummond zum Wiedereintritt einen hochrangigen Sekretariatsposten durchzusetzen, jedoch ebenfalls ohne Erfolg (Barros 1979: 395). Brasilien trat aus, aber Spanien widerrief sein Austrittsgesuch 1928 und trat danach erst 1939 aus. Mexiko kündigte kurz nach dem Beitritt 1932 an wieder auszutreten, widerrief dies allerdings 1933 (Barros 1979: 226-227). Klare nationalistische Gründe wurden bei den Austritten von Nazi-Deutschland und Japan angeführt. Letzteres blieb allerdings Mitglied von Unter-Organisationen des

18 Strollo, Philipp J.: League of Nations Chronology (http://worldatwar.net/timeline/other/le ague18-46.html; 23.8.2019).

19 Art. 1 der Völkerbundsatzung: “Any Member of the League may, after two years' notice of its intention so to do, withdraw from the League, provided that all its international obligations and all its obligations under this Covenant shall have been fulfilled at the time of its withdrawal.". 
Völkerbunds. Mit Machtantritt Mussolinis war ein Austritt Italiens omnipräsent, wurde aber erst 1937 vollzogen (Barros 1979: 392). Ab Mitte der 1930er Jahre war der Völkerbund mit einer Austrittswelle in Lateinamerika konfrontiert. Zuerst Paraguay (1935), dann Guatemala, Honduras, Nicaragua (1936) und El Salvador (1937) sowie Chile und Venezuela (1938) und schließlich Peru (1939). Diese Austritte waren u.a. Ausdruck des Protests von Seiten der lateinamerikanischen Staaten, dass die Monroe-Doktrin in den Völkerbundvertrag integriert worden war (Barros 1979: 215-216).

Neben diplomatischen Verhandlungen blieb dem Völkerbund laut Art. 1 der Völkerbundsatzung ein potenzieller Sanktionsmechanismus, für den Fall, dass der austretende Mitgliedsstaat seinen Verpflichtungen nicht nachgekommen war (Magliveras 1991). Dies beinhaltete nicht nur die finanziellen Beiträge, sondern auch die rechtlichen Verpflichtungen, wie z.B. den Minderheitenschutz. Mögliche Sanktionierungen Nazi-Deutschlands auf dieser Grundlage wurden jedoch nicht vollzogen (Magliveras 1991). Im Fall von Spanien und Brasilien unternahm Generalsekretär Drummond erhebliche diplomatische Anstrengungen, um diese beiden Länder zum Verbleib und, im Fall Brasiliens, zum Wiedereintritt zu überreden (Barros 1979: 119). Drummond überzeugte den Rat an beide Länder zu appellieren im Völkerbund zu verbleiben und zog in Erwägung, zumindest Brasilien im Fall eines Austritts weiterhin an technischer und anderweitiger Kooperation teilhaben zu lassen. Wie in der aktuellen Diskussion um den Brexit rief dies jedoch starke Bedenken hervor, da eine derartige selektive Kooperation einen wichtigen Präzedenzfall für andere Austrittswillige schaffen würde (Barros 1979: 242). Über all diesen diplomatischen Bemühungen des Generalsekretärs lag jedoch die Einsicht, dass ausgetretene Staaten nicht zurückzugewinnen seien: „Brazil will not be won by the League or the Powers running after her“ (Barros 1979: 238).

\section{Zusammenfassung: Herausforderungen und Reaktionen am Beispiel des Völkerbunds}

Der Fall des Völkerbunds illustriert deutlich die Vielfalt der Herausforderungen einer IO durch ihre Mitgliedsstaaten. Bis auf Mandatsbeschränkungen unternahmen Staaten alle Typen der geschilderten Handlungen, wenn auch aus unterschiedlichen Gründen. Erste Einblicke in die Reaktionen auf diese Herausforderungen zeigen, dass der Völkerbund darauf hauptsächlich durch Nicht-Reaktion und die Vermeidung von Öffentlichkeit und nur in den wenigsten Fällen durch Anpassung oder Resilienz-Bildung reagierte, auch wenn institutionelle Mechanismen für letzteres durchaus gegeben waren. Die oben geschilderten Prozesse deuten darauf hin, dass neben der Rolle des Generalsekretärs auch die übrigen Mitgliedsstaaten, insbesondere die Großmächte, für die Varianz in Reaktionen relevant sind. Der Generalsekretär übte hinsichtlich der diplomatischen Verhandlungsführung und der Initiierung möglicher Reaktionen großen Einfluss aus, während sich die Mitgliedsstaaten im Rat für das Fehlen konkreter Sanktionen und damit das Scheitern von Resilienz- 
bildung verantwortlich zeichnen. Allerdings bleibt unklar, welche Faktoren systematisch welche Reaktionen begünstigen. Abschließend sollen deshalb im Folgenden erste Überlegungen zur Erklärung von Reaktionsmustern vorgestellt werden.

\section{Annahmen über die Varianz in den Reaktionen von IOs}

Die Frage, unter welchen Bedingungen IOs in welcher Form auf die Einschränkung ihrer Autorität reagieren, war bislang noch nicht Bestandteil systematischer Forschung. Verschiedene Faktoren können die Reaktionen von IOs beeinflussen, unter anderem die Art der Herausforderung aber auch spezifische institutionelle Charakteristika. Nicht alle oben geschilderten Herausforderungen beeinträchtigen die Autorität von IOs in derselben Weise. Je nachdem, wie unmittelbar sich die Herausforderungen auf die Handlungsfähigkeit auswirken, desto höher ist auch die Bedrohung und desto akuter der Reaktionsbedarf aus Sicht der IO. Budgetkürzungen und Austritte haben unmittelbare Auswirkungen auf die Handlungsfähigkeit, während Personalblockaden und Mandatsbeschränkungen mittelfristig und die systematische Nicht-Einhaltung zentraler Normen eher langfristig wirken.

Des Weiteren ist anzunehmen, dass die Reaktion von IOs unterschiedlich ausfällt, je nachdem welcher Mitgliedsstaat diese Handlungen ausübt. Auch wenn alle Mitgliedsstaaten nach dem Völkerrecht souverän gleichgestellt sind, gibt es doch zentrale Unterschiede was deren Machtpositionen innerhalb von IOs betrifft. Die Verwundbarkeit einer IO gegenüber dem herausfordernden Staat ist hierbei entscheidend. Mächtige Staaten besitzen ein Vetorecht in zentralen Entscheidungen oder dominieren die Entscheidungsfindung aufgrund ihrer relativ hohen Anteile an Mitgliedsbeiträgen, während schwache Staaten kein Vetorecht besitzen und vergleichsweise wenig finanzielle Beiträge leisten. Auf der Basis der Prinzipal-Agent-Literatur ist anzunehmen, dass IOs gegenüber Staaten mit Vetorecht und hohen Mitgliedsbeiträgen verwundbarer sind (Hawkins et al. 2006; Newman et al. 2006).

Schließlich kann erwartet werden, dass IOs, abhängig von ihrem institutionellen Design, unterschiedlich auf Herausforderungen durch Mitgliedsstaaten reagieren. Die Forschung zu internationalen Gerichten zeigt, dass spezifische institutionelle Charakteristika den Gerichten helfen mit herausfordernden Staaten umzugehen (Soley/Steininger 2018). Darüber hinaus analysierte die IO-Forschung, dass die Autorität von IOs sowie die Art der Beitragserhebung beeinflussen, wie IOs gegenüber ihren Mitgliedsstaaten auftreten (Hooghe et al. 2017; Johnson 2014; Lake 2007). Die Autorität von IOs lässt sich unterscheiden, je nachdem wie hoch der Grad der Delegation und wie unabhängig die Bürokratie ist. Die Art der Beitragserhebung variiert zwischen eher unabhängigen, festgesetzten Beiträgen und eher abhängigen, freiwilligen Beiträgen (Haftel/Thompson 2006). Während die meisten IB-Theorien den Fokus auf das Verhalten der anderen Mitgliedsstaaten legen würden, plädiere ich in diesem Beitrag deshalb dafür, auch organisationstheoretische Ansätze für die Erklärung von Reaktionsmustern mit einzubeziehen, um der Komplexität von IOs 
gerecht zu werden. Unter welchen Bedingungen welche Art von Reaktionstyp zu erwarten ist, soll im Folgenden abschließend diskutiert werden.

Gemäß der Literatur zu policy inaction ist inertia insbesondere zu erwarten, wenn die Kosten für ein Handeln im Vergleich mit Nichthandeln hoch sind, wenn die Konsequenzen des Handelns unklar sind oder wenn vergangene Handlungen eine Reaktion blockieren (Rosenschöld et al. 2014). Des Weiteren ist Nichtreaktion zu erwarten, wenn Koalitionen von Mitgliedsstaaten sich gegenseitig blockieren oder wenn bestimmte gatekeepers innerhalb der Mitgliedsstaaten oder der Bürokratie ein Handeln verhindern (McConnell/'t Hart 2019: 647). Nichtreaktion kann sowohl ein Ergebnis einer strategischen Entscheidung sein als auch durch strukturelle, institutionelle Defizite wie z.B. sogenannten blind spots erzeugt werden (McConnell/'t Hart 2019: 654; Bach/Wegrich 2019). Im Zusammenhang mit der Rückforderung nationalstaatlicher Souveränität ist inertia deshalb unter folgenden Bedingungen zu erwarten: erstens, wenn der herausfordernde Staat vergleichsweise wenig Macht besitzt; zweitens, wenn die Art der Herausforderung keine unmittelbare Auswirkung auf die Handlungsfähigkeit der IO hat; und drittens, wenn die übrigen Mitgliedsstaaten keine homogene Position hinsichtlich des Umgangs mit den Herausforderungen einnehmen.

Hunkering ist ein Reaktionstyp, der auftritt, wenn eine oder mehrere sogenannte peer-IOs von demselben Mitgliedsstaat unter Druck gesetzt werden. Die Bedingungen hierfür sind teilweise ähnlich wie für inertia, mit dem Unterschied, dass der herausfordernde Staat aus der Perspektive der IO mächtig genug ist, um sein Verhalten potenziell auf andere IOs zu übertragen, und dass die Herausforderung direkte institutionelle Auswirkungen auf die IO haben würde. Die Literatur zu contested multilateralism verweist ebenfalls darauf, dass die Glaubwürdigkeit des herausfordernden Staates notwendig ist, um eine Reaktion von IOs zu erzeugen (Morse/ Keohane 2014). Abgesehen davon ist hunkering wie inertia dann zu erwarten, wenn die übrigen Mitgliedsstaaten keine homogene Position hinsichtlich des Umgangs mit den Herausforderungen einnehmen oder die Autorität der IO-Bürokratie funktional und finanziell relativ unabhängig ist.

Im Gegensatz zu inertia und hunkering sind adaptation und resilience zwei Reaktionstypen, die für IOs viel kostenintensiver sind. Deshalb sind adaptation und resilience eher zu erwarten, wenn die Maßnahme zur Rückforderung nationalstaatlicher Souveränität erhebliche Auswirkungen auf die Handlungsfähigkeit der IO hat. Hierbei ist weiterhin zu unterscheiden, dass adaptation eher als kurzfristige Reaktion auf erhebliche, unmittelbare Auswirkungen zu erwarten ist, während resilience eine längerfristige Antwort auf erhebliche, aber weniger unmittelbare Herausforderungen darstellt. Auf der Basis der Forschung zu IO-Autorität (Hooghe et al. 2017) ist darüber hinaus zu erwarten, dass finanziell unabhängigere IOs mit supranationaler, regelsetzender Autorität eher resilience ausbilden. Im Gegensatz da$\mathrm{zu}$ ist bei finanziell abhängigeren IOs mit intergouvernementaler und funktionaler Autorität eher adaptation zu erwarten. Die Unabhängigkeit einer IO lässt sich dabei sowohl anhand des Ausmaßes an Delegation festsetzen als auch hinsichtlich der Frage, inwieweit die Mitgliedsbeiträge auf festgesetzten oder freiwilligen Beiträgen 
beruhen (Haftel/Thompson 2006; Hooghe et al. 2017; Lake 2007). Abgesehen von diesen institutionellen Charakteristika sind sowohl für adaptation als auch für resilience auch die Positionen der übrigen Mitgliedsstaaten relevant. Beide Reaktionstypen sind nur zu erwarten, wenn die übrigen Mitgliedsstaaten eine hinreichend homogene Position gegenüber dem herausfordernden Staat einnehmen, um eine Reaktion der IO-Bürokratie nicht zu blockieren.

Zusammenfassend lässt sich sagen, dass die Art der Reaktion von IOs auf die Rückforderung nationalstaatlicher Souveränität von vier Faktoren abhängt: erstens, inwiefern die Herausforderung die Handlungsfähigkeit der IO unmittelbar einschränkt; zweitens, ob der herausfordernde Mitgliedsstaat eine einflussreiche Position innerhalb der IO innehat; drittens, inwieweit die übrigen Mitgliedsstaaten eine homogene Position einnehmen; und viertens, inwieweit die IO einen hohen Grad an Autorität und finanzieller Unabhängigkeit besitzt. Es ist deshalb zu erwarten, dass IOs auf Herausforderungen durch institutionell mächtige Staaten eher durch Anpassung reagieren, während institutionelle Resilienz, die Vermeidung von öffentlicher Sichtbarkeit oder Nicht-Reaktion eher in Reaktion auf Herausforderungen durch institutionell schwächere Staaten geschieht. Inertia und hunkering sind darüber hinaus zu erwarten, wenn die Positionen der übrigen Mitgliedsstaaten nicht homogen sind, während eine homogene Position sowohl für adaptation als auch für resilience notwendig ist. Schließlich ist anzunehmen, dass supranationalere, regelsetzende und finanziell unabhängigere IOs eher dazu tendieren, in Reaktion auf die Herausforderung durch Mitgliedsstaaten institutionelle Resilienz zu bilden oder nicht zu reagieren. Hingegen kann angenommen werden, dass intergouvernementalere, funktionalere und finanziell abhängigere IOs eher mit hunkering oder adaptation reagieren.

\section{Schlussbetrachtungen}

Das Ziel dieses Beitrags war, eine neue Forschungsagenda aufzuzeigen, die das ambivalente Verhältnis zwischen IOs und ihren Mitgliedsstaaten, und damit auch die Einschränkung der Autorität von IOs durch Mitgliedsstaaten, die ihre Souveränität zurückfordern, systematisch beleuchtet. Während die aktuelle Forschung bisher nur auf spezifische Herausforderungen wie z.B. Austritte aus IOs einging, liefert dieser Beitrag einen konzeptionellen Rahmen, um die verschiedenen historischen und aktuellen Herausforderungen an IOs durch ihre Mitgliedsstaaten vergleichend zu analysieren. Diese Typologie umfasst Budgetkürzungen, Personalblockaden, Mandatsbeschränkungen, systematische Normverletzungen und Austritte. Des Weiteren entwickelte der Beitrag eine Typologie zur Analyse der Reaktionen von IOs, die den Fokus darauf richtet, dass IOs in unterschiedlicher Weise auf diese Herausforderungen reagieren. Diese beiden Typologien wurden am Fallbeispiel des Völkerbunds empirisch illustriert, was die extreme Bandbreite an Herausforderungen an diese IO und an möglichen Reaktionen darauf verdeutlichte. 
Tabelle: $\quad$ Übersicht der Typologien

\begin{tabular}{|l|l|}
\hline Staatliche Herausforderungen & Reaktionen der IO \\
\hline Budgetkürzung & Inertia (Nicht-Reaktion) \\
Personalblockade & Hunkering (Vermeidung von Öffentlichkeit) \\
Mandatsbeschränkung & Anpassung \\
systematische Normverletzung & Resilienz (Koalitionsbildung und Ressourcenstär- \\
Austritt & kung) \\
\hline
\end{tabular}

Je nachdem, wie IO Bürokratien und die übrigen Mitgliedsstaaten reagieren, können multilaterale Krisen, die die Autorität von IOs beeinträchtigen, überwunden werden. Andererseits weisen diese Typologien auch Wege auf, die zu einer Handlungsunfähigkeit oder einer formalen Auflösung von IOs führen können. Mehr als ein Drittel der 561 IOs, die seit 1815 entstanden, haben im Zeitraum bis 2015 aufgehört zu existieren, die Mehrheit davon in den 1930er und 1940er Jahren (Eilstrup-Sangiovanni 2018; Gray 2018). Nach dem Zweiten Weltkrieg sind die Dekaden der 1980er und 2000er diejenigen, in denen die wenigsten IOs aufhörten zu existieren. Als Ergänzung der umfangreichen Literatur zur Entstehung und Entwicklung von IOs können die hier entwickelten Typologien von staatlichen Herausforderungen und IO-Reaktionen deshalb dabei helfen, die Lebensdynamiken von IOs umfassender zu analysieren.

Hieraus ergeben sich weitere Fragen für die zukünftige Forschung. Erstens, welche Arten staatlichen Handelns sind für IOs besonders existenzbedrohend und welche Faktoren begünstigen oder lindern diese Bedrohung? Um die besondere Qualität der aktuellen Krise des Multilateralismus einzuschätzen, ist eine Analyse verschiedener Typen von Herausforderungen unter verschiedenen Bedingungen nötig. Nur ein historischer Vergleich der Reaktionen von IOs, die denselben Herausforderungen ausgesetzt waren, kann aufzeigen, wie multilaterale Kooperation im Rahmen von IOs auch in Zukunft fortbestehen kann. Der Blick auf die verschiedenen Arten staatlichen Handelns, das darauf abzielt die Autorität von IOs einzuschränken, verdeutlicht die ambivalente Rolle von Autonomie und Autorität von IOs. IOs mit hoher Autonomie und Autorität generieren mit höherer Wahrscheinlichkeit Widerstand auf Seiten souveränitätsbedachter Mitgliedsstaaten. Gleichzeitig sind ebendiese IOs doch wahrscheinlich eher in der Lage, diese Herausforderungen durch Resilienzbildung einzuhegen.

Zweitens, welche Arten der Reaktion sind besonders effektiv um einerseits multilaterale Krisen einzuhegen und andererseits aber auch Defizite im bestehenden System von IOs zu überwinden? Im Sinne der in diesem Beitrag entwickelten Hypothesen kann angenommen werden, dass sowohl der Status des herausfordernden Mitgliedsstaats als auch das institutionelle Design der IO die Art der Reaktion beeinflussen. Weitere Analysen sind darüber hinaus nötig, die der Frage nachgehen, welche Herausforderungen gleichzeitig auch Chancen auf institutionelle Reform und neue Vitalität, z.B. aufgrund des Austritts von blockierenden Veto-Spielern, bieten. Der Austritt Großbritanniens aus der EU kann dabei einerseits als Autori- 
tätsverlust der EU im Sinne des Einflussbereichs der Organisation gesehen werden, andererseits aber auch als Chance auf eine Verständigung einer Kompetenzvertiefung verstanden werden. Dies eröffnet ein neues Forschungsfeld zur Effektivität der unterschiedlichen Reaktionen von IOs, um zentrale Bedingungen für die Fortexistenz, die Lähmung oder den Untergang von IOs herauszufiltern.

Drittens, welche Rolle spielen Souveränitätskonzeptionen bei der Herausforderung von IOs durch einzelne Mitgliedsstaaten? Das Fallbeispiel des Völkerbunds zeigt, dass Austritte, Personalblockaden oder Budgetkürzungen nicht allein aus Souveränitätserwägungen, sondern aus vielfältigen Gründen unternommen werden. Mit Blick auf die Reden der StaatenvertreterInnen in der jährlichen Versammlung des Internationale Strafgerichtshofs wird deutlich, dass die Verwendung des Begriffs Souveränität stark variiert: im Zeitraum von 2006-2018 wird Souveränität von StaatenvertreterInnen insgesamt 17-mal erwähnt, davon allerdings in den Jahren 2015-2018 allein 14-mal. Davor wird das Konzept nur je einmal in den Jahren 2006, 2012 und 2013 verwendet. Dabei wird Souveränität sowohl als Ausdruck und Bestätigung multilateraler Kooperation als auch als Begründung für Widerstand gegen den Gerichtshof verwendet. Dies weist darauf hin, dass der Begriff deutlichen Popularitätsschwankungen unterliegt und die Gründe für staatliche Herausforderungen im Sinne der oben entwickelten Typologie vielfältiger sind.

Die Gründe, weshalb Staaten die Autorität von IOs einschränken, könnten darüber hinaus einen Einfluss darauf haben, wie effektiv eine IO auf unterschiedliche Herausforderungen reagiert. Allerdings ist dies mehr eine empirische denn theoretische Frage, da aus theoretischer Sicht alle Reaktionen möglich sind. Eine IO kann zahlungsunwilligen und zahlungsunfähigen Mitgliedsstaaten gleichermaßen begegnen, jedoch hätte dieselbe Reaktion deutlich unterschiedliche Auswirkungen auf die Kooperationsbereitschaft dieser Staaten. Kontrafaktisch könnte man argumentieren, dass der Völkerbund wahrscheinlich effektiver seinem Untergang hätte entgegenwirken können, wenn er auf die Austritte von Deutschland und Japan (aufgrund von revisionistischer Motivation) anders reagiert hätte als auf die Androhung eines Austritts durch Brasilien, das dadurch einen Sitz im Rat verhandeln wollte. Es bleibt daher Aufgabe der zukünftigen Forschung zu untersuchen, welche unterschiedlichen Effekte eine bestimmte Art der Reaktion hat, je nachdem wie die jeweiligen Herausforderungen begründet sind.

Anliegen dieses Beitrags war es, das Verhalten von Staaten gegenüber IOs und deren Reaktionen darauf systematischer zu analysieren und damit verschiedene Handlungsoptionen aufzuzeigen. Mit dem konzeptionellen Fokus auf IO-Bürokratien plädiert der Beitrag dafür, IOs in ihrer Komplexität auch theoretisch besser zu erfassen. Die IO-Forschung selbst steht aufgrund der eingangs erwähnten Gräben zwischen pro- und anti-multilateral eingestellten Staaten vor neuen Herausforderungen. Erst die Zukunft wird zeigen, welche dieser beiden Strömungen das Zusammenleben von Staaten dominieren wird und was dies für die Autorität von IOs bedeutet. 


\section{Literatur}

Abbott, Kenneth W./Genschel, Philipp/Snidal, Duncan/Zangl, Bernhard (Hrsg.). 2015. International Organizations as Orchestrators. Cambridge: Cambridge University Press.

Abbott, Kenneth W./Snidal, Duncan 1998: Why States Act through Formal International Organizations. Journal of Conflict Resolution, 42: 1, 3-32.

Alter, Karen J./Gathii, James T./Helfer, Laurence R. 2016: Backlash against International Courts in West, East and Southern Africa: Causes and Consequences. European Journal of International Law, 27: 2, 293-328.

Ashworth, Lucian M. 2011. Realism and the Spirit of 1919: Halford Mackinder, Geopolitics and the Reality of the League of Nations. European Journal of International Relations, 17: 2, 279-301.

Bach, Tobias/Kai Wegrich, Kai (Hrsg.) 2019. The Blind Spots of Public Bureaucracy and the Politics of Non-Coordination. Cham: Palgrave Macmillan/ Springer.

Barnett, Michael/Finnemore, Martha 2004: Rules for the World. International Organizations in Global Politics. Ithaca: Cornell University Press.

Barros, James 1979: Office without power: Secretary-General Sir Eric Drummond 1919-1933. Oxford: Clarendon Press.

Bauer, Michael W./Ege, Jörn 2016: Bureaucratic Autonomy of International Organizations' Secretariats. Journal of European Public Policy, 23: 7, 1019-1037.

Biermann, Rafael 2017: The Role of International Bureaucracies. In Rafael Biermann \& Joachim A. Koops (Hrsg.) The Palgrave Handbook of inter-organizational relations in world politics (243-270). Basingstoke: Palgrave Macmillan.

Borzyskowski, Inken von/Vabulas, Felicity 2019: Hello, Goodbye: When do States Withdraw from International Organizations? Review of International Organizations, 14: 2, 335366.

Brölmann, Catherine/Collins, Richard/Droubi, Sufyan/Wessel, Ramses A. 2018: Exiting International Organizations. A Brief Introduction. International Organisations Law Review, 15: 2, 243-263.

Chayes, Abram/Handler Chayes, Antonia 1995: The New Sovereignty: Compliance with International Regulatory Agreements. Cambridge MA, London: Harvard University Press.

da Conceição-Heldt, Eugénia 2016: Verantwortungszurechnung im EU- Mehrebenensystem während der Eurokrise: Wer kontrolliert die Troika- Institutionen? Zeitschrift für Politikwissenschaften, 26: Supplement 1, 115-129.

Daugirdas, Kristina 2015: Reputation and the Responsibility of International Organizations. European Journal of International Law, 25: 4, 991-1018.

Dijkzeul, Dennis/Beigbeder, Yves (Hrsg.). 2003. Rethinking international organizations: Pathology and promise. New York: Berghahn Books.

Ecker-Ehrhardt, Matthias 2018: International Organizations 'Going Public'? An Event-History Analysis of Public Communication Reforms from 1950 to 2015. International Studies Quarterly, 62: 4, 723-736.

Edwards, Don Agustin 1929: Latin America and the League of Nations. Journal of the Royal Institute of International Affairs, 8: 2, 134-153.

Eilstrup-Sangiovanni, Mette 2018: Death of international organizations. The organizational ecology of intergovernmental organizations, 1815-2015. Review of International Organizations, First Online: https://doi.org/10.1007/s11558-018-9340-5.

Ellis, Charles Howard 1928: The origin, structure and working of the League of Nations. London: George Allen \& Unwin.

Finnemore, Martha/Sikkink, Kathryn 1998: International norm dynamics and political change. International Organization, 52: 4, 887-917.

Fischer-Lescano, Andreas 2014: Human Rights in Times of Austerity Policy. The EU Institutions and the Conclusion of Memoranda of Understanding. Baden-Baden: Nomos. 
Francioni, Francesco 2000: Multilateralism à la Carte: the Limits of Unilateral Withholdings of Assessed Contributions to the UN Budget. European Journal of International Law, 11: $1,43-59$.

Gehring, Thomas/Faude, Benjamin 2014: A Theory of Emerging Order within Institutional Complexes: How Competition Among Regulatory International Institutions Leads to Institutional Adaptation and a Division of Labor. Review of International Organizations, 9: 4, 471-498.

Graham, Erin R. 2014: International organizations as collective agents: Fragmentation and the limits of principal control at the World Health Organization. European Journal of International Relations, 20: 2, 366-390.

Graham, Erin R. 2015: Money and multilateralism: how funding rules constitute IO governance. International Theory, 7: 1, 162-194.

Gray, Julia 2018: Life, Death, or Zombie? The Vitality of International Organizations. International Studies Quarterly, 62: 1, 1-13.

Gray, Julia 2019: How International Organizations Survive. Unv. Manuskript.

Haas, Ernst B. 1990: When Knowledge is Power. Three Models of Change in International Organizations. Berkeley: University of California Press.

Haftel, Yoram Z./Thompson, Alexander 2006: The Independence of International Organizations. Journal of Conflict Resolution, 50: 2, 253-275.

Hawkins, Darren G./Lake, David A./Nielson, Daniel L./Tierney, Michael J. (Hrsg.). 2006. Delegation and Agency in International Organizations. Cambridge: Cambridge University Press.

Helfer, Laurence R. 2005: Exiting Treaties. Virginia Law Review, 91: 7, 1579-1648.

Helfer, Laurence R. 2018: Populism and International Human Rights Institutions: A Survival Guide. University of Copenhagen: iCourts Working Paper Series No. 133.

Hell, Stefan Matthias 2007: Siam and the League of Nations Modernization, Sovereignty, and Multilateral Diplomacy, 1920-1940. Leiden University, Leiden.

Heupel, Monika/Zürn, Michael (Hrsg.) 2017: Protecting the Individual from International Authority. Human Rights in International Organizations. Cambridge: Cambridge University Press.

Hirschmann, Gisela 2012: Peacebuilding in UN Peacekeeping Exit Strategies: Organized Hypocrisy and Institutional Reform. International Peacekeeping, 19: 2, 170-185.

Hirschmann, Gisela 2019: Guarding the guards: Pluralist accountability for human rights violations by international organisations. Review of International Studies, 45: 1, 20-38.

Hirschmann, Gisela 2020: Accountability in Global Governance. Oxford: Oxford University Press.

Hooghe, Liesbet/Marks, Gary/Lenz, Tobias/Bezuijen, Jeanine/Ceka, Besir/ Derderyan, Svet 2017: Measuring International Authority: A Postfunctionalist Theory of Governance. Oxford: Oxford University Press.

Hunter Wade, Robert 2002: US hegemony and the World Bank: the fight over people and ideas. Review of International Political Economy, 9: 201-229.

Ikenberry, G. John 2009: Liberal Internationalism 3.0: America and the Dilemmas of Liberal World Order. Perspectives on Politics, 7: 1, 71-87.

Ikenberry, G. John 2018: The end of liberal international order? International Affairs, 94: 1, $7-23$.

Johnson, Tana 2014: Organizational Progeny. Why Governments are Losing Control over the Proliferating Structures of Global Governance. Oxford: Oxford University Press.

Jones, Erik 2018: Towards a Theory of Disintegration. Journal of European Public Policy, 25: $3,440-451$.

Karns, Margaret P./Mingst, Karen A. 1990a: Continuity and Change in U.S. -IGO Relationships: A Comparative Analysis With Implications for the Future of Multilateralism in U.S. Foreign Policy. In Margaret P. Karns \& Karen. A. Mingst (Hrsg.) The United States 


\section{Aufsätze}

and Multilateral Institutions. Patterns of Changing Instrumentality and Influence (289-319). Boston: Unwin Hyman.

Karns, Margaret P./Mingst, Karen A. (Hrsg.). 1990b. The United States and Multilateral Institutions. Patterns of Changing Instrumentality and Influence. Boston: Unwin Hyman.

Keefer, Edward C. 2005: The Nixon Administration and the United Nations: "It's a Damned Debating Society". Paper presented at the 8e Conférence internationales des pays éditeurs de documents diplomatiques, 6-7 Octobre 2005, Paris.

Koremenos, Barbara 2016: The Continent of International Law: Explaining Agreement Design. Cambridge: Cambridge University Press.

Koremenos, Barbara/Lipson, Charles/Snidal, Duncan 2001: The rational design of international institutions. International Organization, 55: 4, 761-799.

Kraske, Joachim/Becker, William H./Diamond, William/Galambos, Louis 1996: Bankers with a Mission. The Presidents of the World Bank 1946-1991. New York/ Washington DC: The World Bank/ Oxford University Press.

Lake, David A. 2007: Delegating divisible sovereignty: Sweeping a conceptual minefield. Review of International Organizations, 2, 219-237.

League of Nations 1930: Ten Years of World Co-operation. Geneva: Secretariat of the League of Nations.

Lipson, Michael 2007: Peacekeeping: Organized Hypocrisy? European Journal of International Relations, 13: 1, 5-34.

Luck, Edward C. 1999: Mixed Messages: American Politics and International Organization Washington, DC: Brookings Institution Press.

Magen, Amichai 2016: Cracks in the Foundations: Understanding the Great Rule of Law Debate in the EU. Journal of Common Market Studies, 54: 5, 1050-1061.

Magliveras, Konstantin D. 1991: The Withdrawal From the League of Nations Revisited. Dickinson Journal of International Law, 10: 1, 25-71.

Malone, David M./Kong, Yuen Foong (Hrsg.). 2003. Unilateralism and U.S. Foreign Policy. International Perspectives. Boulder, London: Lynne Rienner.

McConnell, Allan/'t Hart, Paul 2019. Inaction and Public Policy: Understanding Why Policymakers 'Do Nothing'. Policy Sciences, 52: 4, 645-661.

Morse, Julia C./Keohane, Robert O. 2014: Contested multilateralism Review of International Organizations, 9: 4, 385-412.

Newman, Edward/Thakur, Ramesh/Tirman, John (Hrsg.). 2006. Multilateralism under challenge? Power, international order, and structural change. Tokyo, New York: United Nations University Press.

Park, Susan 2018: International Organizations and Global Problems. Theories and Explanations. Cambridge: Cambridge University Press.

Pauwelyn, Joost/Hamilton, Rebecca J. 2018: Exit from International Tribunals. Journal of International Dispute Settlement, 9: 4, 679-690.

Pedersen, Susan 2007: Back to the League of Nations. The American Historical Review, 112: 4, 1091-1117.

Pedersen, Susan 2015: The Guardians: The League of Nations and the Crisis of Empire. Oxford: Oxford University Press.

Reinalda, Bob/Verbeek, Bertjan (Hrsg.). 2004. Decision Making within International Organizations. London and New York: Routledge.

Rosenschöld, Johan Munck af/ Rozema, Jaap G./ Frye-Levine, Laura Alex 2014. Institutional Inertia and Climate Change: A Review of the New Institutionalist Literature. WIREs Climate Change, 5, 639-648.

Sarooshi, Danesh 2005: International Organizations and Their Exercise of Sovereign Powers. Oxford: Oxford University Press.

Seibel, Wolfgang 2015: Politisierung internationaler Organisationen: Eine theoretische Einordnung am Beispiel der Vereinten Nationen und des Prinzips der Schutzverantwortung. In Eugénia da Conceicao-Heldt, et al. (Hrsg.) Internationale Organisationen: Autonomie, 
Politisierung, interorganisationale Beziehungen und Wandel. PVS-Sonderheft 49 (244 270). Baden-Baden: Nomos.

Snidal, Duncan 1985. Coordination versus prisoners' dilemma: Implications for international cooperation and regimes. American Political Science Review, 79, 923-942.

Soley, Ximena/Steininger, Silvia 2018: Parting ways or lashing back? Withdrawals, backlash and the Inter-American Court of Human Rights. International Journal of Law in Context, 14: Special Issue 2, 237-257.

Stahn, Carsten/El Zeidy, Mohamed M. (Hrsg.) 2011. The International Criminal Court and Complementarity: From Theory to Practice. Cambridge: Cambridge University Press.

Stein, Arthur 1983. Coordination and collaboration: Regimes in an anarchic world. In Stephen Krasner (Hrsg.) International regimes (115-140). Ithaca, NY: Cornell University Press.

Tallberg, Jonas/Sommerer, Thomas/Squatrito, Teresa/Jönsson, Krister 2013: The Opening Up of International Organizations. Transnational Access in Global Governance. Cambridge: Cambridge University Press.

Tallberg, Jonas/Zürn, Michael 2019: The legitimacy and legitimation of international organizations: Introduction and framework. Review of International Organizations, Online First: https://doi.org/10.1007/s11558-018-9330-7.

Taylor, Amos E. 1925: Economic Sanctions and International Security. University of Pennsylvania Law Review, 74: 2, 155-168.

Webber, Douglas 2014: How Likely Is It That the European Union Will Disintegrate? A Critical Analysis of Competing Theoretical Perspectives. European Journal of International Relations, 20: 2, 341-365.

Wehrli, Yannick 2015: New Histories of Latin America at the League of Nations. In A. MacPherson \& Y. Wehrli (Hrsg.) Beyond geopolitics : New histories of latin america at the league of nations. (1-18). Albuquerque: University of New Mexico Press.

Wouters, Jan/Odermatt, Jed 2016: Assessing the Legality of Decisions. In J. K. Cogan, et al. (Hrsg.) The Oxford Handbook of International Organizations (Ch. 47). Oxford: Oxford University Press.

Zangl, Bernhard/Heußner, Frederick/Kruck, Andreas/Lanzendörfer, Xenia 2016: Imperfect adaptation: how the WTO and the IMF adjust to shifting power distributions among their members. Review of International Organizations, 11: 2, 171-196.

Zürn, Michael 2018a: Contested Global Governance. Global Policy, 9: 1, 138-145.

Zürn, Michael 2018b: A Theory of Global Governance. Oxford: Oxford University Press.

Zürn, Michael/Binder, Martin/Ecker-Ehrhardt, Matthias 2012: International authority and its politicization. International Theory, 4: 1, 69-106.

Zürn, Michael/Deitelhoff, Nicole 2015: Internationalization and the State: Sovereignty as the External Side of Modern Statehood. In S. Leibfried, et al. (Hrsg.) The Oxford Handbook of Transformations of the State (193-217). New York, NY: Oxford University Press.

Zürn, Michael/Ecker-Ehrhardt, Matthias (Hrsg.). 2013. Die Politisierung der Weltpolitik. Umkämpfte internationale Institutionen. Berlin: Edition Suhrkamp. 